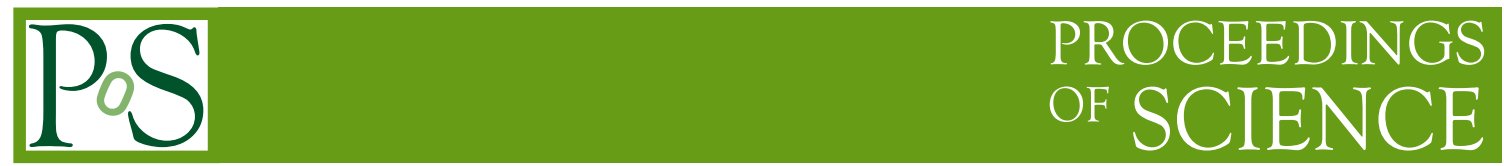

\title{
Hard X-ray surveys and the local AGN population
}

\section{Sergey Sazonov*}

Space Research Institute, Russian Academy of Sciences

Moscow, Russia

E-mail: sazonovaiki.rssi.ru

We discuss some recent achievements in our understanding of the local AGN population based on large-area hard X-ray surveys (in particular, by the IBIS instrument aboard INTEGRAL), focusing on the issue of the fraction of obscured AGN as a function of hard X-ray luminosity.

11th INTEGRAL Conference Gamma-Ray Astrophysics in Multi-Wavelength Perspective, 10-14 October 2016

Amsterdam, The Netherlands

${ }^{*}$ Speaker. 


\section{Introduction}

Hard X-ray (at energies above $\sim 10 \mathrm{keV}$ ) surveys allow one to obtain a nearly unbiased census of unobscured and obscured AGN (except for Compton thick objects, which are relatively difficult to detect even in hard X-rays). Although there were space-borne hard X-ray detectors long ago, their sensitivity and/or field of view were not sufficient to collect good statistics of AGN in the local Universe (let alone the more distant Universe). The situation changed in 2004, when the results of the RXTE slew survey were reported [9], which had covered the entire extragalactic sky $\left(|b|>10^{\circ}\right)$ in the 3-20 keV band with a record sensitivity of $\sim 10^{-11} \mathrm{erg} \mathrm{s}^{-1} \mathrm{~cm}^{-2}$. Using these data, we [10] detected $\sim 100$ AGN and studied a number of key properties of the local $(z<0.1)$ AGN population, namely the luminosity function and the luminosity dependence of the number ratio of obscured and unobscured AGN [10]. Soon thereafter, the first results of observations by the IBIS and BAT coded-mask instruments aboard the INTEGRAL and Swift spacecraft, respectively, appeared. These missions have provided the first sensitive all-sky surveys at energies above $15 \mathrm{keV}$ and finally opened the era of extragalactic hard X-ray surveys.

Over the years, hard X-ray source catalogs compiled from IBIS and BAT data have greatly increased in size and their latest versions include $\sim 400[2,6]$ and $\sim 700$ [1] AGN, respectively. This is more than sufficient for undertaking statistical studies of the local AGN population, provided that complementary information about the distances, X-ray absorption column densities and other properties of these objects is available. Such information has been accumulating over the years thanks to the X-ray and optical follow-up efforts of several teams, but usually a few years pass after release of a given hard X-ray catalog before it achieves high completeness in terms of soft $\mathrm{X}$-ray and optical information.

\section{Fraction of obscured AGN as a function of hard X-ray luminosity}

We recently [12] made use of a highly complete (in terms of optical identification and X-ray absorption information) sample of 151 non-blazar AGN located at $|b|<5^{\circ}$, selected in the 17-60 keV energy band from the INTEGRAL/IBIS 7-year (December 2002-July 2009) all-sky hard Xray survey [5], to investigate if the declining trend of the fraction of obscured AGN with increasing luminosity, observed in many studies, is mostly an intrinsic or selection effect. The sample comprises 67 unobscured (X-ray column density $N_{\mathrm{H}}<10^{22} \mathrm{~cm}^{-2}$ ) and 84 obscured $\left(N_{\mathrm{H}} \geq 10^{22} \mathrm{~cm}^{-2}\right.$ ) AGN, including 17 heavily obscured $\left(N_{\mathrm{H}} \geq 10^{24} \mathrm{~cm}^{-2}\right)$ ones. For most of these Compton thick (or nearly Compton thick) objects, there are now reliable $N_{\mathrm{H}}$ estimates based on high-quality NuSTAR spectroscopic data.

Using a radiative transfer model based on torus obscuration geometry, we demonstrated that in addition to a negative bias in finding heavily obscured AGN in hard X-ray flux limited surveys there must also exist a positive bias in detecting unobscured AGN - due to reflection by the torus of part of the radiation emitted by the central source toward the observer. Since the AGN luminosity function steepens at high luminosities, these observational biases must inevitably lead to a decreasing observed fraction of obscured AGN with increasing luminosity even if this fraction has no intrinsic luminosity dependence. 
We explored two possibilities for the central hard X-ray source in AGN: (i) isotropic emission (as is assumed in most studies) and (ii) emission collimated according to Lambert's law, $d L / d \Omega \propto \cos \alpha$, where $\alpha$ is the angle with respect to the axis of the torus (which is a fairly natural assumption in the context of disk-corona geometry). In the former case, the intrinsic (i.e. corrected for the biases discussed above) obscured AGN fraction reconstructed from our sample shows a declining trend with increasing luminosity, although the inferred intrinsic obscured fraction proves to be larger than the observed one. Namely, the obscured fraction is larger than $\sim 85 \%$ at $L \lesssim 10^{42.5} \mathrm{erg} \mathrm{s}^{-1}$ and decreases to $\lesssim 60 \%$ at $L \gtrsim 10^{44} \mathrm{erg} \mathrm{s}^{-1}(17-60 \mathrm{keV})$. In terms of the half-opening angle $\theta$ of the torus, this implies that $\theta \lesssim 30^{\circ}$ in lower luminosity AGN, and $\theta \gtrsim 45^{\circ}$ in higher luminosity ones. If, however, the emission from the central SMBH is collimated, then the derived intrinsic dependence of the obscured AGN fraction is consistent with the opening angle of the torus being constant with luminosity, namely $\theta \sim 30^{\circ}$ (see Fig. 1).
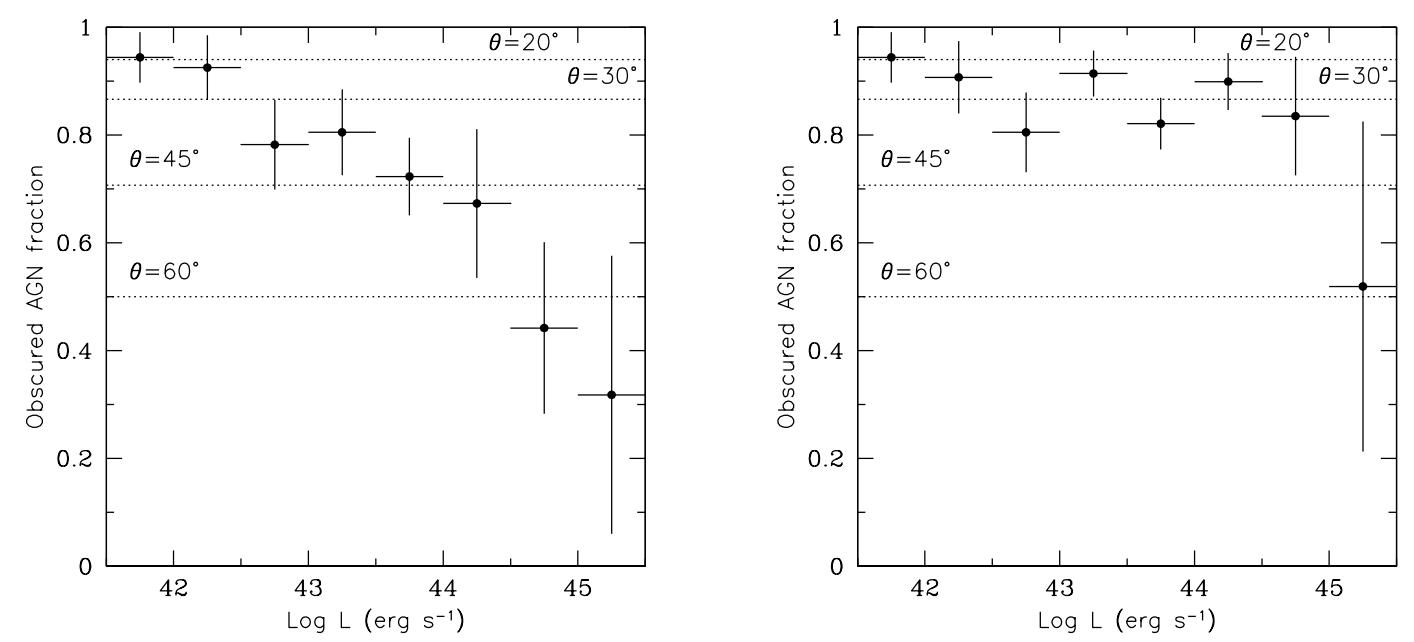

Figure 1: Left: Reconstructed intrinsic fraction of obscured AGN as a function of intrinsic hard X-ray (17$60 \mathrm{keV}$ ) luminosity for a torus half-opening angle of $30^{\circ}$ and an isotropic central source. Right: The same but for collimated emission $(d L / d \Omega \propto \cos \alpha)$ from the central source. The dotted lines indicate the fraction of the sky obscured from the central source by a torus with half-opening angle $\theta=20^{\circ}, 30^{\circ}, 45^{\circ}$ or $60^{\circ}$. Adapted from [12].

We regard both possiblities - the intrinsic obscuring AGN fraction declining with luminosity or being constant - as feasible, as the angular emission diagram of the central source in AGN is poorly known. A more careful comparison of these and other existing estimates of the ratio of obscured and unobscured AGN in future work may help get insight into the geometrical and physical properties of obscuration in AGN, which may be different in X-ray, optical, infrared and radio bands. Our constraints on the intrinsic dependence of the obscured AGN fraction on luminosity can be improved using larger samples of hard X-ray selected AGN from INTEGRAL, Swift and NuSTAR surveys. However, it will be practially impossible to improve the current, fairly uncertain estimate of the obscured AGN fraction at the highest luminosities $\left(\gtrsim 10^{45} \mathrm{erg} \mathrm{s}^{-1}\right)$ in the local Universe, since the INTEGRAL and Swift all-sky surveys are sensitive enough to detect all such objects in the local $(z \lesssim 0.2)$ Universe and have found just a few of them. 
We have also reconstructed the intrinsic (corrected for the biases discussed above) hard Xray luminosity functions of local unobscured and obscured AGN (see Fig. 2) and estimated the total number density and luminosity density of AGN with $L>10^{40.5} \mathrm{erg} \mathrm{s}^{-1}$ [12]. These accurate local measurements may be used as reference $z=0$ values in studying cosmic AGN evolution and modeling the cosmic X-ray background.
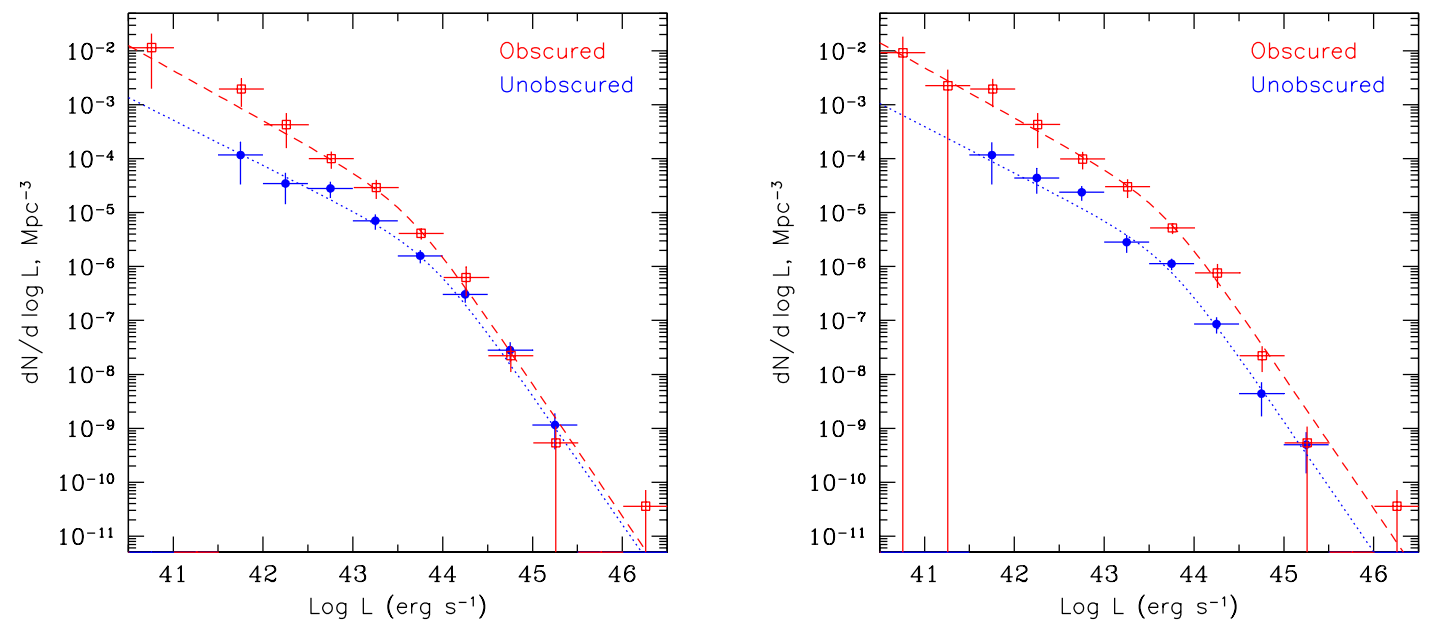

Figure 2: Left: Intrinsic hard X-ray (17-60 keV) luminosity functions of unobscured (blue filled circles) and obscured (red empty squares) AGN, fitted by broken power laws (blue dotted line and red dashed line, respectively), reconstructed under the assumption of a torus half-opening angle of $30^{\circ}$ and an isotropic central source. Right: The same but for the case of collimated emission $(d L / d \Omega \propto \cos \alpha)$ from the central source. Adapted from [12].

\section{Deep INTEGRAL extragalactic surveys}

The observational program of INTEGRAL has been mainly dedicated to Galactic source studies, whereas the high Galactic latitude sky has been observed less intensively and very inhomogeneously. However, a number of multi-year campaigns have been performed in the extragalactic sky, in particular in the 3C 273/Coma region [8], around the Large Magellanic Cloud [4] and most recently around the M81 galaxy [11,3]. Importantly, the sensitivity of the IBIS instrument in these extragalactic fields continues to grow nearly proportionally to the square root of exposure showing no significant contribution of systematic noise. In combination with IBIS large field of view, this opens up a possibility to collect a significantly large sample of hard X-ray emitting AGN with fluxes down to a few $10^{-12} \mathrm{erg} \mathrm{s}^{-1} \mathrm{~cm}^{-2}$. Such objects, due to their rarity in the sky, evade NuSTAR deep surveys.

We have recently presented [7] results of a deep survey of three extragalactic fields, M81 (dead time corrected exposure of 9.7 Ms), LMC (6.8 Ms) and 3C 273/Coma (9.3 Ms), in the hard X-ray (17-60 keV) energy band with the IBIS telescope, based on 12 years of observations (2003-2015). The combined survey reaches a $4 \sigma$ peak sensitivity of $0.18 \mathrm{mCrab}\left(2.6 \times 10^{-12} \mathrm{erg} \mathrm{s}^{-1} \mathrm{~cm}^{-2}\right)$ and sensitivity better than 0.25 and 0.87 mCrab over $10 \%$ and $90 \%$ of its full area of $4900 \mathrm{deg}^{2}$, 
respectively. We have detected in total 147 sources at $S / N>4 \sigma$, including 37 sources observed in hard X-rays for the first time (see Fig. 3). The survey is dominated by AGN (98 identified objects of this type). The nature of 25 sources remains unknown.
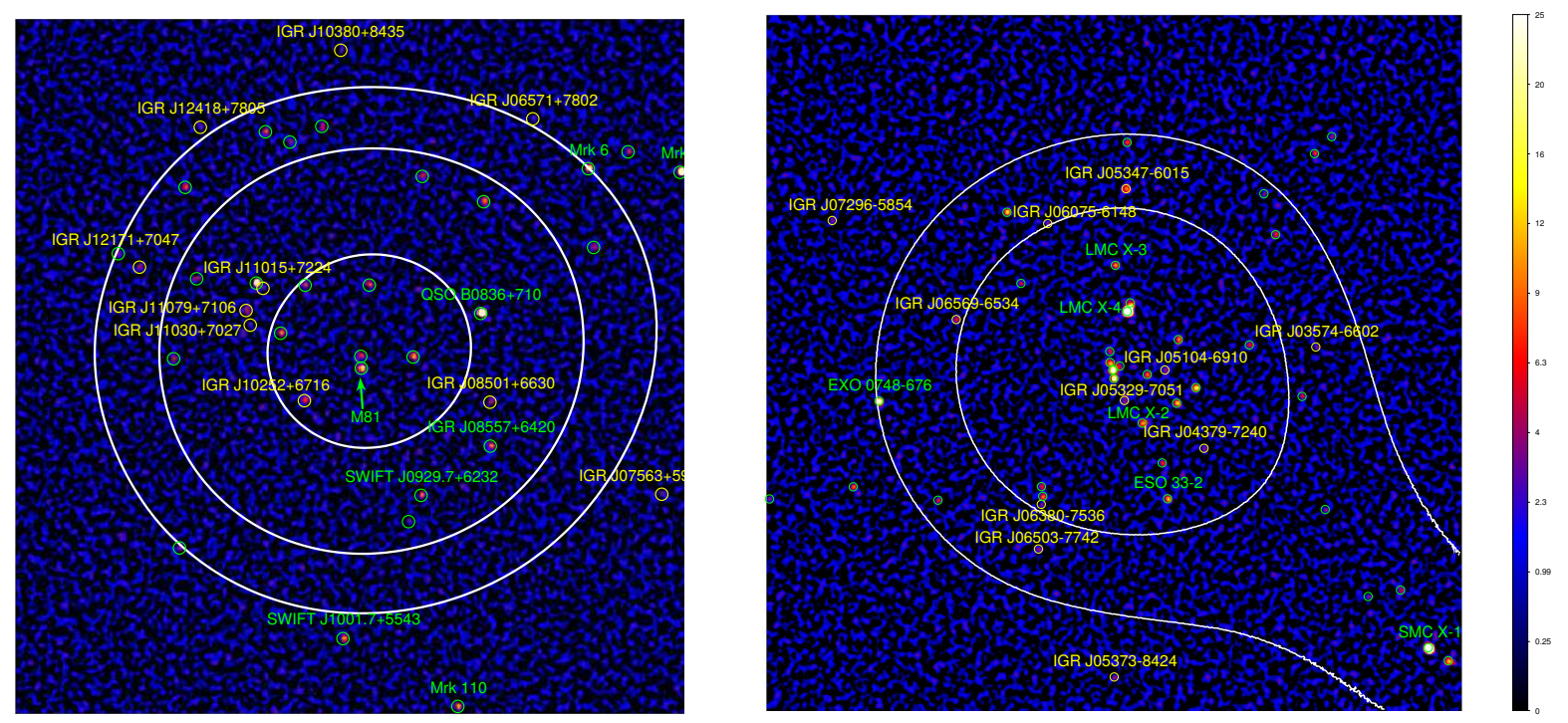

Figure 3: Hard X-ray maps of the M81 and LMC fields, shown in terms of significance. Yellow circles denote new sources and green circles already known ones. Some of the brightest sources are marked for easy navigation. Left: The M81 field. The peak exposure $9.7 \mathrm{Ms}$, contours show exposures of 2, 4 and 8 Ms. Right: The LMC field. The peak exposure $6.8 \mathrm{Ms}$, contours drawn at 2 and 4 Ms. Adapted from [7].

Using these data, we constructed AGN number-flux relations $(\log N-\log S)$ and calculated AGN number densities in the local Universe for the entire survey and for each of the three extragalactic fields down to fluxes $\sim 3 \times 10^{-12} \mathrm{erg} \mathrm{s}^{-1} \mathrm{~cm}^{-2}$, which is deeper by a factor of two compared to previous (all-sky) measurements. The AGN number counts for the M81 and 3C 273/Coma fields are consistent with each other, while the LMC field demonstrates a steeper number-flux distribution ( $2 \sigma$ deviation from the expected $-3 / 2$ slope) and a lack of bright AGN with flux higher than $2 \times 10^{-11} \mathrm{erg} \mathrm{s}^{-1} \mathrm{~cm}^{-2}$.

\section{References}

[1] Baumgartner W. H., Tueller J., Markwardt C. B., Skinner G. K., Barthelmy S., Mushotzky R. F., Evans P. A., Gehrels N., 2013, ApJS, 207, 19

[2] Bird A. J., et al., 2016, ApJS, 223, 15

[3] Churazov E., et al., 2014, Natur, 512, 406

[4] Grebenev S. A., Lutovinov A. A., Tsygankov S. S., Winkler C., 2012, Natur, 490, 373

[5] Krivonos R., Tsygankov S., Revnivtsev M., Grebenev S., Churazov E., Sunyaev R., 2010, A\&A, 523, AA61 
[6] Krivonos R., Tsygankov S., Lutovinov A., Revnivtsev M., Churazov E., Sunyaev R., 2012, A\&A, 545, A27

[7] Mereminskiy I. A., Krivonos R. A., Lutovinov A. A., Sazonov S. Y., Revnivtsev M. G., Sunyaev R. A., 2016, MNRAS, 459, 140

[8] Paltani S., Walter R., McHardy I. M., Dwelly T., Steiner C., Courvoisier T. J.-L., 2008, A\&A, 485, 707

[9] Revnivtsev M., Sazonov S., Jahoda K., Gilfanov M., 2004, A\&A, 418, 927

[10] Sazonov S. Y., Revnivtsev M. G., 2004, A\&A, 423, 469

[11] Sazonov S. Y., Lutovinov A. A., Krivonos R. A., 2014, AstL, 40, 65

[12] Sazonov S., Churazov E., Krivonos R., 2015, MNRAS, 454, 1202 